\title{
MINIMAL RATES OF HEAD GROWTH IN THE FIRST FOUR MONTHS OF LIFE
}

\author{
BY \\ EILEEN M. O'NEILL \\ From the Department of Paediatrics, City General Hospital, Sheffield
}

(RECEIVED FOR PUBLICATION JANUARY 25, 1962)

In a previous publication (O'Neill, 1961) it was shown how abnormally rapid head growth could be detected quite simply by means of control lines and the construction and significance of these lines were given. The present paper is concerned with the same method but the control lines represent minimum normal rates of head growth.

\section{Material and Methods}

The normal head circumference values of the previous survey (O'Neill, 1961) were used. Included in the survey were 676 full-term and 225 premature babies.

Full details of the method can be obtained by reference to the earlier publication mentioned above. For the following more brief explanation an example showing a stage in the making of the tenth week control lines is given in Fig. 1. Here the head circumference values of all the normal children at 10 weeks of age coincide at the point $\mathrm{X}$. The rates of head growth of each child from birth to 10 weeks are represented by curves drawn backwards from this point and the heavy lines represent the limits of normal. If an abnormal growth curve is added to this graph, in the same way, it will be found to lie outside these boundaries. If above the upper limit head growth will have been abnormally slow up to this age and if below the lower limit growth will have been abnormally rapid during this same period of time. In this way the boundaries act as control lines. The upper one, as we have seen, represents the minimum normal rate of head growth from birth to 10 weeks and is known as the tenth week 'minimum' control line. It is with this control line that we are interested in the present paper.

In order to avoid confusion the lower boundary, which represents the maximum normal rate of head growth up to 10 weeks and which was described previously (O'Neill, 1961) will, in future, be called the 'maximum' control line for the tenth week.

Graphs similar to those described above were

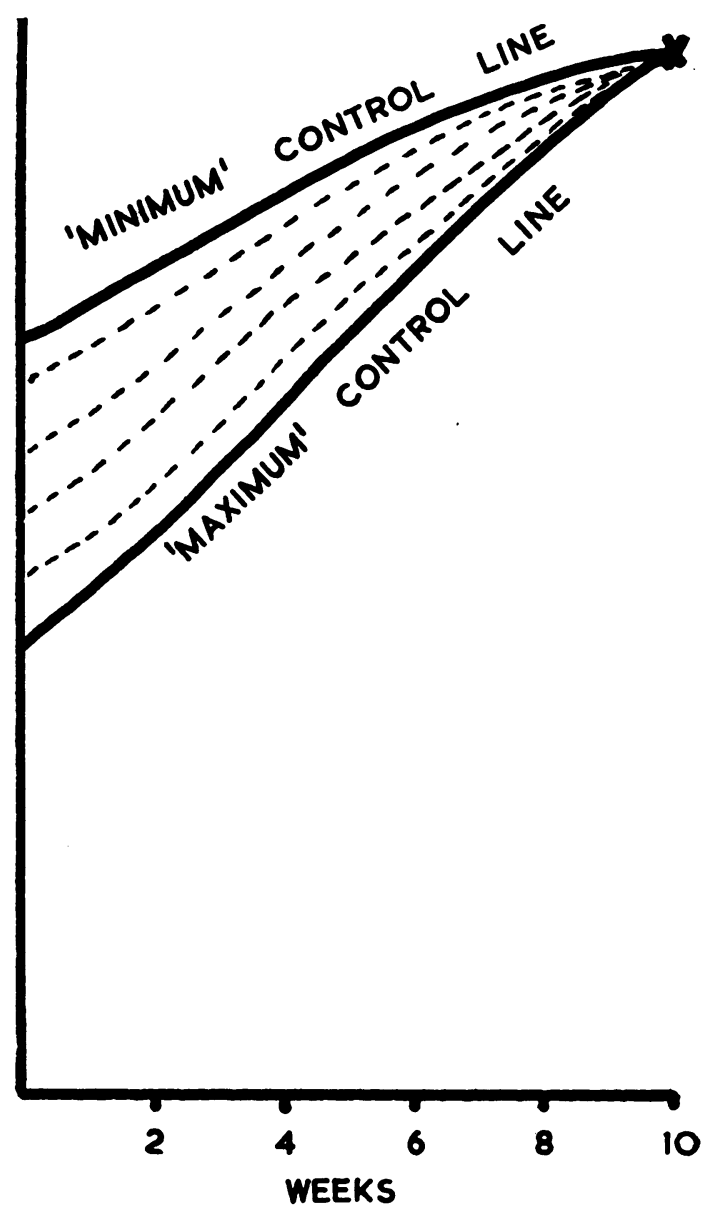

FIG. 1.-Diagram to demonstrate construction of tenth week control lines. Broken lines represent the normal head circumference curves; heavy black lines the limits of normal. The upper boundary shows the minimum rate of increase in head circumference and the lower one the maximum rate of increase in head circumference consistent with normality. The former is known as the tenth week 'minimum' control line and the latter as the tenth week 'maximum' control line. 


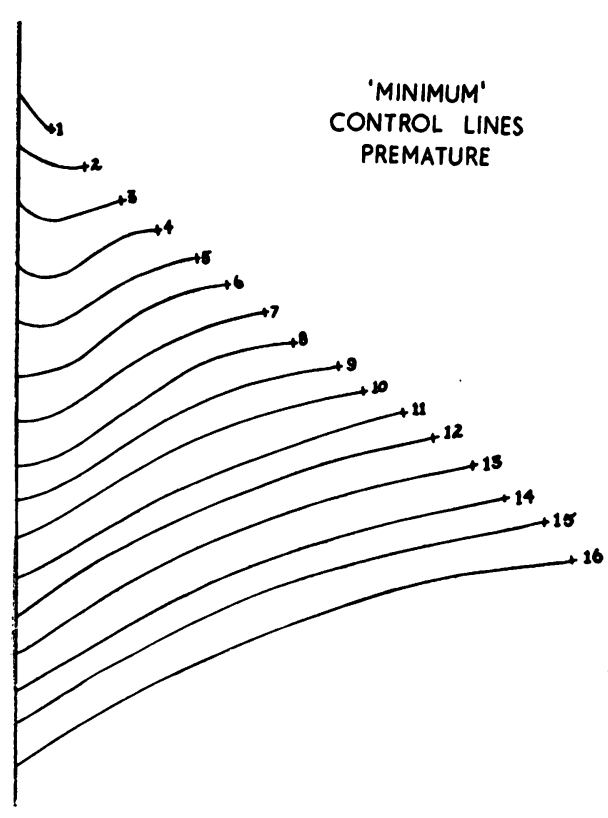

Fig. 2.-Control lines: premature infants. Each line represents the minimum normal rate of increase in head circumference from birth to the week of age indicated by the number at the end of the line.

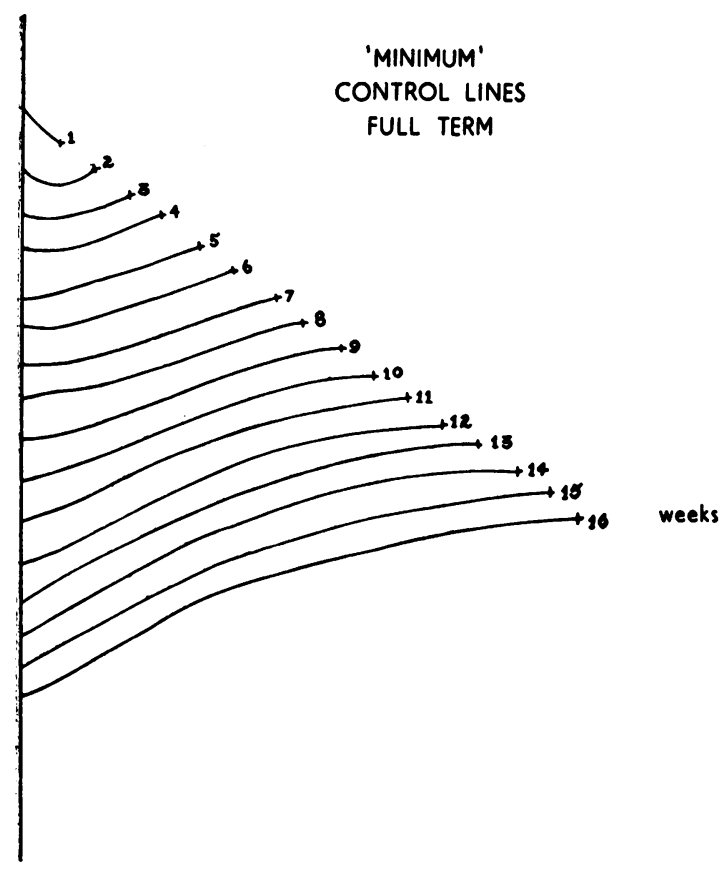

FIG. 3.-Control lines: full-term infants. Each line represents the minimum rate of increase in head circumference from birth to the week of age indicated by the number at the end of the line.

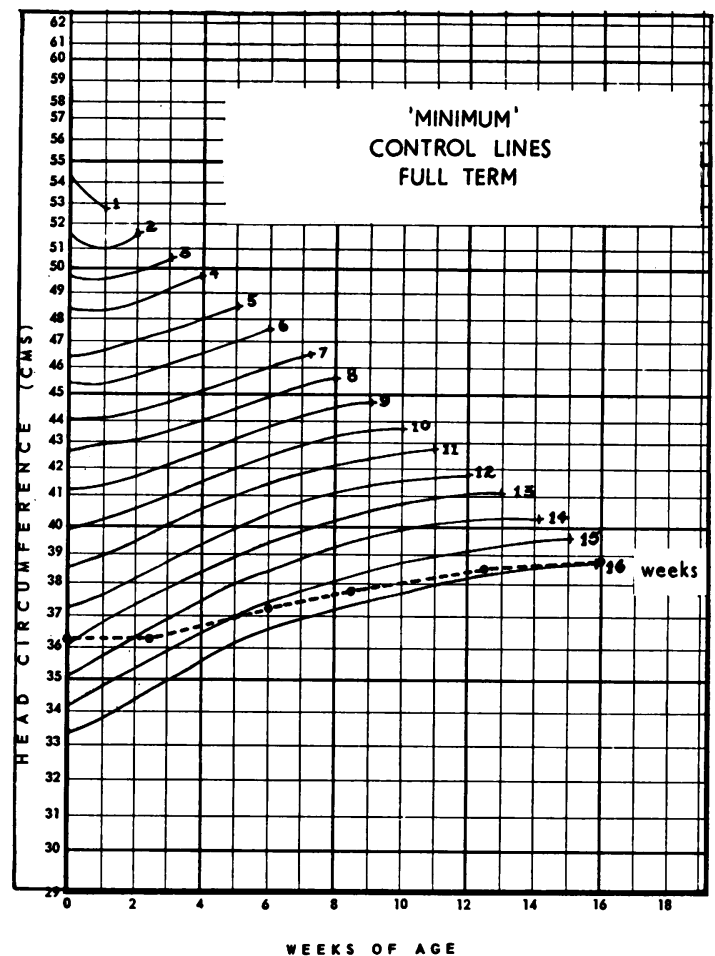

Fig. 4. - Control lines superimposed on a ratio chart. - . - . line joining actual head circumference values. The control lines are placed in the position used to assess the rate of head growth at the age of 16 weeks in a full-term baby. The line joining the head circumference values lies above the sixteenth week control line, and therefore head growth between birth and 16 weeks has been less than normal.

made for each week from 1 to 16 weeks and from these the 'minimum' control lines for the premature babies were grouped together as shown in Fig. 2 . A similar set of 'minimum' control lines was made for the full-term infants (Fig. 3). Both sets are drawn on transparent paper.

\section{How to Use 'Minimum' Control Lines}

1. Plot the patient's head circumference values, in centimetres, on a ratio chart (O'Neill, 1961) and join the points.

2. Over the ratio chart place the appropriate set of 'minimum' control lines (premature or full-term) and adjust so that the ordinate of the control lines accurately overlies that of the ratio chart whilst, at the same time, the $\mathrm{X}$ at the end of the appropriate control line coincides with the last head circumference measurement. An example is given in Fig. 4 and shows the end of the sixteenth week 
control line superimposed on the sixteenth week head measurement.

3. Now examine the position of the head circumference curve which was drawn on the ratio chart. If it lies either partly or wholly above the 'minimum' control line, of the week in question, then head growth has been abnormally slow. If, on the other hand, the head circumference curve lies completely below the 'minimum' control line, then head growth has been normal or greater than normal up to this age.

\section{Discussion}

The reason for carrying out the original normal head circumference survey has already been discussed (O'Neill, 1961). The values for minimum rates of head growth from which the 'minimum' control lines were obtained were incidental to the previous work. However, they are reported here because minimum rates of growth obtained from such detailed material have not, to my knowledge, been published before.

To avoid confusion it should be appreciated that the normal and abnormal head circumference curves relative to the control lines are reversed if one considers maximum instead of minimum rates of growth, or vice versa. Thus, using 'minimum' control lines the abnormal head circumference curve would lie above the control line but using 'maximum' control lines the abnormal head circumference curve would be found below the control line. This is easy to remember if one interprets the findings in terms of 'steepness' of the curves.

Suggested uses for the 'minimum' control lines are the study of all types of microcephaly presenting at, or shortly after, birth; the possible detection of transient abnormally slow rates of head growth not leading to established microcephaly, and the follow-up of anoxic newborn babies, many of the worst of whom become microcephalic. The method could also be used as a guide to the approximate time of onset of cerebral damage. For example, if a newborn infant's head circumference is at the 75th percentile for the birth weight but its head growth from birth is abnormally slow it could be reasonably concluded that cerebral damage took place shortly before or just after birth. In some cases this may seem obvious, but more often than not it is difficult to know whether neonatal anoxia was the result or cause of cerebral abnormality.

\section{Summary}

A method of diagnosing abnormally slow rates of head circumference growth by means of 'minimum' control lines and ratio charts is described.

'Minimum' control lines for each week from 1 to 16 weeks are given for both full-term and premature babies.

It is suggested that they may be useful in the study of microcephaly and cerebral anoxia.

I wish to thank Dr. R. R. Gordon for his valuable criticism and advice; also Mrs. W. Wright for constructing the control lines from the data obtained during the head growth survey.

REFERENCE

O'Neill, E. M. (1961). Normal head growth and the prediction of head size in infantile hydrocephalus. Arch. Dis. Childh., 36, 241 . 